\title{
Left Hepatic Artery Pseudoaneurysm Caused by Acute Pancreatitis
}

\author{
Hwa Seong Nam ${ }^{a}$ Myung Hwan Noh ${ }^{a}$ Ji Eun Han ${ }^{a}$ Jae Hoon Kimª \\ Ki Jong Oha Hyuk Lee ${ }^{a}$ Jeong Hyun Job \\ Departments of ${ }^{\mathrm{a}}$ Internal Medicine and ${ }^{\mathrm{b}}$ Radiology, College of Medicine, Dong-A University, \\ Busan, Republic of Korea
}

\section{Significance of the Study}

- This rare case of left hepatic artery pseudoaneurysm, which occurred following severe acute pancreatitis, was successfully treated with coil embolization.

- Awareness of this clinical manifestation could be important for early diagnosis and treatment.

\section{Keywords}

Pseudoaneurysm · Left hepatic artery · Acute pancreatitis · Endoscopic papillectomy

\section{Abstract}

Objective: The aim of this work was to report a case of left hepatic artery pseudoaneurysm due to acute pancreatitis following endoscopic papillectomy. Clinical Presentation and Intervention: A 74-year-old female with an ampullary adenoma underwent papillectomy, which was complicated by acute pancreatitis. Computed tomography showed aneurysmal dilatation of the proximal left hepatic artery. An angiography with coli embolization was performed and was successful. The patient was doing well at the 1-year follow-up. Conclusion: This patient with left hepatic artery pseudoaneurysm following severe acute pancreatitis was successfully treated with coil embolization.

(c) 2017 S. Karger AG, Basel
\end{abstract}

\section{KARGER}

E-Mail karger@karger.com www.karger.com/mpp

\section{(c) 2017 S. Karger AG, Basel}

This is an Open Access article licensed under the terms of the Creative Commons Attribution-NonCommercial 3.0 Unported license (CC BY-NC) (www.karger.com/OA-license), applicable to the online version of the article only. Distribution permitted for non-commercial purposes only.

\section{Introduction}

Hepatic artery pseudoaneurysm (HAP) is a rare complication of acute or chronic surgical injury to the hepatic artery. Sethi et al. [1] reported that $19 \%$ of pseudoaneurysms due to pancreatitis occur in the hepatic artery. The HAP usually occurs in the common or proper hepatic arteries rather than the peripheral arterial branches. As a result, most HAPs occur in the extrahepatic region $[1,2]$. While there are some data regarding the prevalence and treatment of pseudoaneurysms in chronic pancreatitis, very little has been reported in acute pancreatitis [3, 4]. Right HAP caused by acute pancreatitis has been reported sporadically $[4,5]$, but left HAP caused by acute pancreatitis has not been reported. Hence, we hereby report the successful treatment of a left HAP with coil embolization. 
Fig. 1. Abdominal CT scan findings. a The first day of admission (before endoscopic papillectomy). b After endoscopic papillectomy, showing aneurysmal dilatation of the left hepatic artery in the fissure for ligamentum venosum (arrow, late arterial phase).

Fig. 2. Angiography displaying the fusiform aneurysmal dilatation of the left hepatic artery (arrow; a) and the finding of embolization with microcoils (b).
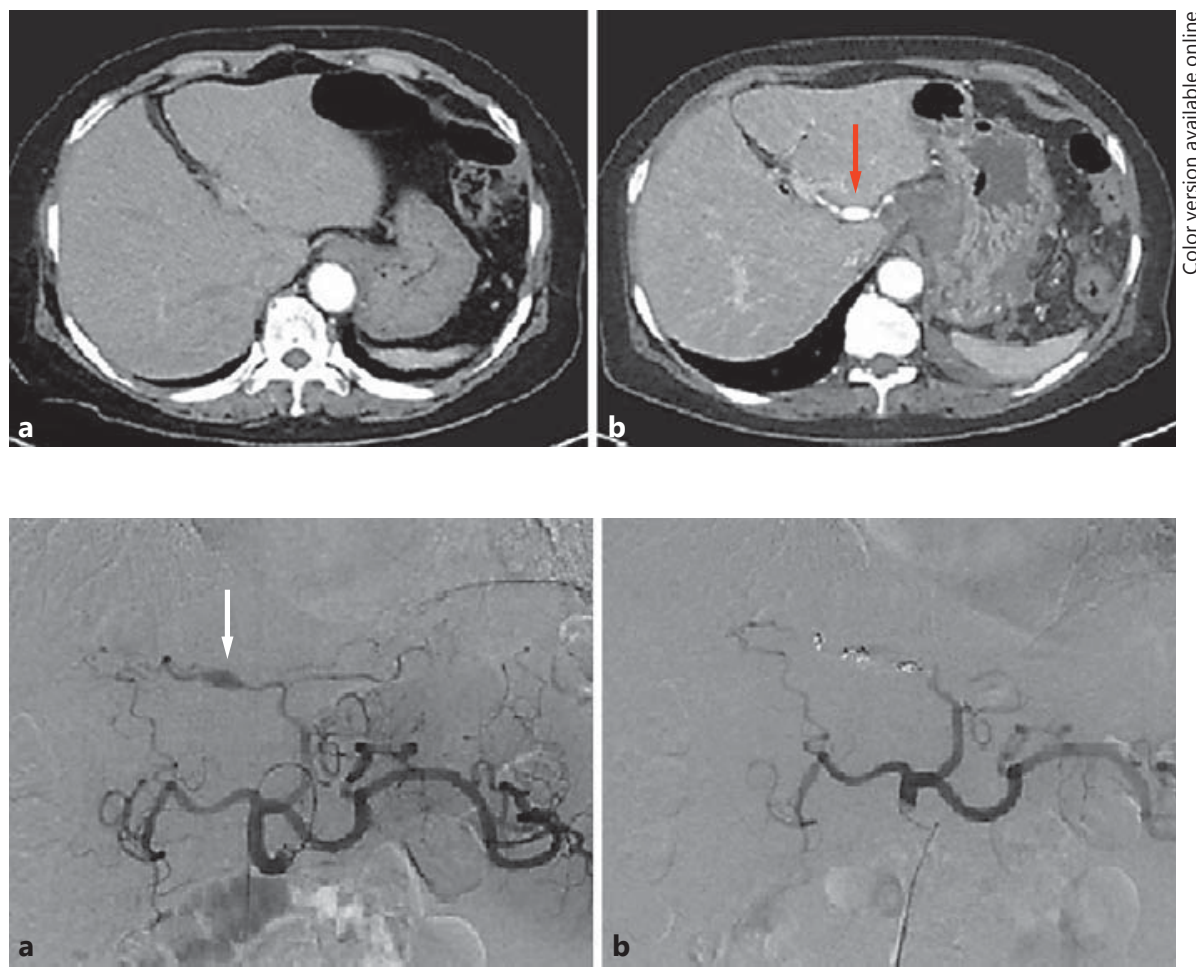

\section{Case Report}

A 74-year-old female with a history of hypertension was admitted for an endoscopic papillectomy to remove an ampullary adenoma with low-grade dysplasia which was proven by previous biopsy. Her vital signs were stable and a physical examination revealed unremarkable findings. An abdominal computed tomography (CT) scan upon admission day showed unremarkable findings (Fig. 1a). The blood test results upon admission were white blood cell count (WBC) 5,640/ $\mu \mathrm{L}$, hemoglobin $13.2 \mathrm{~g} / \mathrm{dL}$, and platelet count $299,000 / \mu \mathrm{L}$. Liver function tests were all within normal limits (aspartate aminotransferase $40 \mathrm{IU} / \mathrm{L}$, alanine aminotransferase $36 \mathrm{IU} / \mathrm{L}$ ) and so was the normal serum total bilirubin level $(0.4 \mathrm{mg} / \mathrm{dL})$. Serology was negative for hepatitis B and hepatitis $\mathrm{C}$, and serum amylase and lipase, the renal function test, and clotting profiles were also within normal limits. C-reactive protein was $0.07 \mathrm{mg} / \mathrm{dL}$ and the serum level of CA 19-9 was below $2 \mathrm{U} / \mathrm{mL}$ (normal 0-37 U/mL). We performed endoscopic papillectomy on the day after admission, with endoscopic retrograde cholangiopancreatography (ERCP) showing a slight bulging of the ampulla of Vater and normal bile duct figures. After performing a papillectomy, the pancreatic duct was accessed and the injection of contrast showed good drainage, based on which we did not insert a pancreatic duct stent. During the ERCP, we did not use a guidewire or catheter that might injure the left hepatic artery. On the day following the endoscopic papillectomy, epigastric and periumbilical abdominal pain developed and $\mathrm{WBC}(10,960 / \mu \mathrm{L})$ and serum amylase (1,868 IU/L; normal 42-116) and lipase (3,775 U/L; normal 1-60) were increased. The patient was diagnosed with acute pancreatitis on the basis of her clinical findings and laboratory data.
An abdominal CT scan 3 days after the papillectomy revealed acute pancreatitis with peripancreatic fat infiltration and fluid collections. The symptoms of acute pancreatitis and elevated WBC and serum amylase and lipase lasted for several days. The abdominal CT scan was rechecked 9 days after the papillectomy, revealing severe acute pancreatitis with necrosis and fat infiltration and a newly developed aneurysmal dilatation $(1.5 \times 1.0 \mathrm{~cm})$ of the proximal portion of the left hepatic artery in the fissure for ligamentum venosum (Fig. 1b). On day 6 after the detection of the aneurysm by abdominal CT scan (15 days after the endoscopic papillectomy), we performed angiography and embolization using a sandwich method with microcoils for a fusiform aneurysm on the left hepatic artery (Fig. 2). After embolization, the abdominal CT scan showed well-embolization state of aneurysmal dilatation of the left hepatic artery. One week later, embolization, serum amylase and lipase levels were further decreased, and almost all of the subjective symptoms had subsided. The patient received continued supportive care for acute pancreatitis and was discharged on the 39th hospital day. She had an uneventful course during 1 year of follow-up.

\section{Discussion}

This case of HAP was a result of acute pancreatitis that occurred following endoscopic papillectomy and involved an intrahepatic branch of the left hepatic artery, which is an unusual site. Embolization with angi- 
ography was successfully performed just after the detection of the HAP.

HAP rupture is common and occurs in up to $76 \%$ [6]. Thus, early diagnosis and treatment are considered to be essential. Intrahepatic HAPs account for only about $20 \%$ of all HAPs and most of them arise from a complication of percutaneous procedures such as transhepatic cholangiography, transhepatic drainage catheter placement, or liver biopsy [7]. Therefore, acute pancreatitis could be considered a rare cause of intrahepatic pseudoaneurysm. According to the analysis of 7 cases of HAP reported by Finley et al. [5], 4 developed in the common hepatic artery in the extrahepatic region, 1 was in the right hepatic artery in the extrahepatic region, and 2 cases were developed in the intrahepatic branch of right hepatic artery. A recent report of HAP by Kazue et al. [4] also reported a pseudoaneurysm that involved the right hepatic artery as a complication of acute pancreatitis. Our case of HAP was a result of acute pancreatitis, but it occurred in the early phase following papillectomy, which is very rare, and the involvement of an intrahepatic branch of the left hepatic artery is also unusual. The most sensitive test for detecting HAP is selective angiography. A pseudoaneurysm usually develops 3-5 weeks after the onset of acute pancreatitis, but pseudoaneurysm hemorrhage may occur from a few days to several years after the onset of pancreatitis $[1,6]$. In our patient, the pseudoaneurysm of the left hepatic artery may have occurred during the early phase of inflammation and was detected only 9 days after the onset of acute pancreatitis. Pancreatic duct stents and/or postprocedure rectal nonsteroidal anti-inflammatory drug suppositories should be utilized to lower the risk of severe post-ERCP pancreatitis (PEP) in high-risk patients [8]. Some meta-analyses report that placement of prophylactic pancreatic stents may lower the overall incidence of PEP in high-risk patients, but not the incidence of severe PEP [9]. Furthermore, some debate still exists regarding the use of indomethacin [10]. In this case, we did not use rectal indomethacin and a pancreatic duct stent, which might not alter the process of severe inflammation in acute pancreatitis. It is our opinion that severe acute pancreatitis could be a rapid development of pseudoaneurysm at an unusual remote site, such as the left intrahepatic region. For the management of an arterial pseudoaneurysm arising in the setting of pancreatitis, coil embolization should be initially attempted if the patient is hemodynamically stable [2].

\section{Conclusion}

This patient with a left HAP occurring following severe acute pancreatitis was successfully treated with a coil embolization.

\section{References}

1 Sethi H, Peddu P, Prachalias A, et al: Selective embolization for bleeding visceral artery pseudoaneurysms in patients with pancreatitis. Hepatobiliary Pancreat Dis Int 2010;9: 634-638.

2 Siegelman SS, Copeland BE, Saba GP, et al: CT of fluid collections associated with pancreatitis. AJR Am J Roentgenol 1980;134: 1121-1132.

3 Singh CS, Giri K, Gupta R, et al: Successful management of hepatic artery pseudoaneurysm complicating chronic pancreatitis by stenting. World J Gastroenterol 2006;12: 5733-5734.
4 Kazue S, Manabu W, Takashi I, et al: Right hepatic artery pseudoaneurysm complicating acute pancreatitis. Med Princ Pract 2013;22: 402-404.

5 Finley DS, Hinojosa MW, Paya M, et al: Hepatic artery pseudoaneurysm: a report of seven cases and a review of the literature. Surg Today 2005;35:543-547.

6 Bergert H, Hinterseher I, Kersting S, et al: Management and outcome of hemorrhage due to arterial pseudoaneurysms in pancreatitis. Surgery 2005; 137:323-328.

7 Rösch J, Petersen BD, Hall LD, et al: Interventional treatment of hepatic arterial and venous pathology: a commentary. Cardiovasc Intervent Radiol 1990;13:183-188.
8 Tenner S, Baillie J, DeWitt J, et al: American College of Gastroenterology guideline: management of acute pancreatitis. Am J Gastroenterol 2013;108:1400-1415.

9 Shi QQ, Ning XY, Zhan LL, et al: Placement of prophylactic pancreatic stents to prevent post-endoscopic retrograde cholangiopancreatography pancreatitis in high-risk patients: a meta-analysis. World J Gastroenterol 2014;20:7040-7048.

10 John ML, Stuart RG, Linda LF, et al: Rectal indomethacin does not prevent post-ERCP pancreatitis in consecutive patients. Gastroenterology 2016;150:911-917. 